\title{
Amplitude-Coherent Detection for Optical Wireless Communications: Opportunities and Limitations
}

\section{MOHAMmAD A. AL-JARRAH ${ }^{1}$ (Member, IEEE), ARAFAT AL-DWEIK ${ }^{\circledR 2,3}$ (Senior Member, IEEE), KI-HONG PARK $^{\oplus 4}$ (Senior Member, IEEE), AND MOHAMED-SLIM ALOUINI ${ }^{\oplus 4}$}

\author{
${ }^{1}$ School of Electrical and Electronic Engineering, University of Manchester, Manchester M13 9PL, U.K. \\ ${ }^{2}$ Center for Cyber Physical Systems, Khalifa University, Abu Dhabi, UAE \\ ${ }^{3}$ Department of Electrical and Computer Engineering, Western University, London, ON N6A 3K7, Canada \\ ${ }^{4}$ Computer, Electrical and Mathematical Science and Engineering Division, King Abdullah University of Science and Technology, Thuwal 23955 , Saudi Arabia \\ CORRESPONDING AUTHOR: A. AL-DWEIK (e-mail: arafat.dweik@ku.ac.ae) \\ This work was supported by KU Center for Cyber Physical Systems under Grant C2PS-T2.
}

\begin{abstract}
The proliferation of ubiquitous computing applications created a multi-dimensional optimization problem that includes several conflicting variables such as spectral efficiency, complexity, power consumption, delay, and error probability. To relax the problem and provide efficient solutions, it was necessary to augment the currently overutilized radio spectrum with new frequency bands such as the optical spectrum, which can be used to off-load some of the traffic of certain applications. Therefore, this paper presents an efficient system design that uses amplitude-coherent (AC) detection to reduce the complexity of optical wireless communication systems (OWC), improve its reliability and spectral efficiency. More specifically, we use amplitude shift keying with orthogonal frequency division multiplexing (OFDM) at the transmitter, and AC detection at the receiver. The complexity reduction is achieved by using a low complexity detector, channel estimator, and peak-to-average power ratio (PAPR) reduction scheme. The spectral efficiency is achieved by using real data symbols with discrete cosine transform (DCT), which requires a subcarrier spacing that is 50\% of the discrete Fourier transform (DFT), and does not require Hermitian symmetry to generate real-valued OFDM signals. Moreover, the derived channel estimator is blind, and the PAPR reduction scheme does not require a feedback overhead between the transmitter and receiver.
\end{abstract}

INDEX TERMIS Optical communications, visible light communications, free space optics, OWC, VLC, FSO, DCT-OFDM, PAPR, amplitude-coherent detection, semi-coherent detection.

\section{INTRODUCTION}

$\mathrm{O}$ PTICAL wireless communication (OWC) systems offer some unique features that can make them alternative or complementary to radio frequency (RF) systems. One of the pivotal features is that OWC systems operate in unlicensed frequency band, which is enormously broader than RF bands [1]-[3]. Correspondingly, the license-free ultra-high bandwidth is beneficial to serve ultra-high transmission rates with reasonable cost. For example, Tsonev et al. [4] demonstrated a visible light communications (VLC) system that can offer a data rate of $3 \mathrm{Gbps}$. Free space optics (FSOs) can generally support much higher data rates as demonstrated in [5]. Ultraviolet communication (UVC) data rates are generally much less than VLC and FSO links, particularly for long distances. For example, Puschell and Bayse [6] demonstrated a UVC link with $1.2 \mathrm{Mbps}$ over $1.6 \mathrm{~km}$ distance while the authors in [7] demonstrated an experiment with data rates of $71 \mathrm{Mbps}$ for very short distance such as $8 \mathrm{~cm}$. Due to the high directivity of beam propagation, OWC systems are inherently more immune than RF to particular security threats, and entirely immune to electromagnetic interference.

\section{A. OWC MODULATION SCHEMES}

Although OWC spectrum is in the order of 100s of Terahertz, there are several challenges that limit high data rate transmission, with reasonable cost and complexity. Typically, the 
main limitation for achieving high data rates is the availability of hardware that can support such high rates. In VLC, for example, the modulation response for commercially available LEDs can be as low as few $\mathrm{MHz}$, and with the application of blue filter at the receiver side can increase to $20 \mathrm{MHz}$ [4]. Even with the use of fast LEDs that can have up to $60 \mathrm{MHz}$ bandwidth [4], the utilized bandwidth remains very limited as compared to the available one. Consequently, using spectral and energy efficient modulation schemes remains crucial to design reliable and cost-efficient OWC systems. It is worth noting that the IEEE 802.15.7 standard proposes binary amplitude shift keying (ASK), also denoted as on-off keying (OOK), variable pulse-position modulation (VPPM), and color-shift keying (CSK) as modulation techniques for indoor VLC systems. In this standard the highest data-rate envisaged is $96 \mathrm{Mbps}$ for OOK and CSK, or $24 \mathrm{Mbps}$ for VPPM.

In the literature, a wide range of modulation schemes have been investigated for OWC, a comprehensive survey can be found in [8]. Generally speaking, the considered modulation schemes can be classified as single [9]-[11] or multi-carrier [12]-[17], coherent or noncoherent, multi-level or binary [18]-[23]. A particular combination from these options determines the system spectral efficiency, energy efficiency, complexity, and error rate. Nevertheless, regardless of the selected modulation scheme, the resulted signal should be positive and real when intensity modulation and direct detection (IM-DD) are considered. Using multicarrier techniques such as orthogonal frequency division multiplexing (OFDM) can improve the spectral efficiency of OWC [4], however the energy efficiency is not high due to the peak-to-average power ratio (PAPR) problem, which requires high DC bias values to avoid clipping the negative part of the OFDM signal [12], [13], [17]. Moreover, the spectral efficiency of OFDM when used in OWC with IM-DD is less than what is expected with radio frequency (RF) applications, which is due to the fact that OFDM signals have to be real and positive. For example, in DC biased optical OFDM (DCOOFDM), only $50 \%$ subcarriers may carry information, while it is about $25 \%$ for asymmetrically clipped optical OFDM (ACO-OFDM). The high PAPR may also introduce positive peaks clipping effects as the practical LEDs have the typical nonlinear characteristics. The PAPR problem has been extensively investigated for both RF [24]-[27] and OWC [17], [28]-[31], where the main focus of most researchers is to minimize the PAPR and avoid bit error rate (BER) degradation, while reducing the computational complexity and the required side information.

\section{B. OWC CHANNEL IMPAIRMENTS}

The channel behavior for OWC depends on several factors such as the characteristics of the hardware, distance between the transmitter and receiver, and the environmental conditions. In the literature, the performance of OWC has been investigated using non-fading and fading channel models. The typical fading models are the log-normal,
Gamma-Gamma, Rice, negative exponential, and I-K fading, and additive white Gaussian noise (AWGN) is used to model the receiver noise [8]. The frequency-selectivity and coherence time are important considerations for OWC. For FSO links, the channel delay spread is usually negligible even in severe atmospheric conditions. However, the channel coherence time is very large which implies that the signal might go into a deep fade for periods of time that span thousands of symbols. For VLC, the channel may exhibit frequency selectivity due to the light reflections from walls and other objects [32]. Phase noise and I/Q imbalance are other factors that affect the error performance of OWC systems when coherent modulation schemes are adopted [33].

To recover the information symbols coherently for both $\mathrm{RF}$ and $\mathrm{OWC}$, the receiver generally requires full knowledge of the channel state information (CSI), i.e., amplitude and phase [34], [35]. Therefore, efficient CSI estimation techniques should be adopted at the receiver side. CSI estimation for RF and OWC applications has attracted extensive research efforts as reported in [36]-[40], and the references listed therein. The main objectives of most techniques are to improve the accuracy, reduce the complexity, and avoid using training and pilot signals to increase the spectral efficiency. For AC detection, only the channel amplitude is required to detect the information symbols [34], [35]. Estimating the channel amplitudes in RF applications has been considered in [34], [41]. However, in such techniques it is required to have a hybrid OFDM frame structure that has both amplitude and phase modulated symbols. Consequently, such schemes can not be used for OWC that utilizes the inverse discrete cosine transform (IDCT). Riediger et al. [42] proposed a blind detection scheme that does not require CSI to extract the information symbols. Nevertheless, the proposed scheme is limited to binary OOK using intensity modulation and direct detection (IM/DD).

\section{ASK DETECTION SCHEMES}

Unipolar MASK signals can be typically detected using coherent, noncoherent (NC), or AC detection. The classification of various detection schemes can be performed based on metrics such CSI requirements or BER. Generally speaking, all detectors can be formulated as

$$
\hat{s}=\arg \min _{d_{m}} \mathcal{F}\left(d_{m} \mid \mathrm{CSI}\right)
$$

where $\hat{s}$ is the estimated symbol value, $d_{m}$ is transmitted symbol which is selected uniformly form a particular ASK constellation, and $\mathcal{F}$ is the objective function of the detector. The CSI can be divided into two types, instantaneous (CSI-I), and statistical (CSI-S). Given that the channel coefficient is denoted as $h$, and the additive Gaussian noise (AWGN) is $n$, then CSI-I $\in\{\alpha, \theta\}$, where $\alpha \triangleq|h|$ and $\theta \triangleq \arg \{h\}$. Similarly, CSI-S $\in\left\{\mu_{h}, \sigma_{h}, \sigma_{n}\right\}$, where $\mu_{h} \triangleq|\mathrm{E}[h]|, \sigma_{h}$ is the standard deviation of $h$, and $\sigma_{n}$ is the AWGN standard deviation. 
TABLE 1. Different MASK detection schemes and CSI requirements.

\begin{tabular}{|c|c|c|c|}
\hline Type & $\mathcal{F}$ & CSI-I & CSI-S \\
\hline Coherent $^{\dagger}$ & $\left|r-h d_{m}\right|^{2}$ & $\alpha, \theta$ & - \\
\hline $\mathrm{NC}$ & $-\ln \left(\pi \sigma_{\mathrm{r}}^{2}\right)+\frac{1}{\sigma_{r}^{2}}\left|r-\mu_{h} d_{m}\right|^{2}$ & - & $\sigma_{h}, \mu_{h}, \sigma_{n}$ \\
\hline $\mathrm{AC}-\mathrm{O}$ & $\frac{\alpha^{2}}{2 \sigma_{n}^{2}} d_{m}^{2}-\ln \mathcal{I}_{\theta}$ & $\alpha$ & $\sigma_{h}, \mu_{h}, \sigma_{n}$ \\
\hline AC-S & $\frac{\alpha^{2}}{2 \sigma_{n}^{2}} d_{m}^{2}-\ln \left[I_{0}\left(\frac{\alpha}{\sigma_{n}^{2}} d_{m}|r|\right)\right]$ & $\alpha$ & $\sigma_{n}$ \\
\hline $\mathrm{AC}-\mathrm{H}^{\dagger}$ & {$\left[\frac{|r|^{2}}{\alpha^{2}}-d_{m}^{2}\right]^{2}$} & $\alpha$ & - \\
\hline
\end{tabular}

Table 1 shows the detectors for the coherent, NC, the AC optimum (AC-O), suboptimum (AC-S) and heuristic $(\mathrm{AC}-\mathrm{H})$. Because the detector design depends on the distribution of $h, \mathcal{F}$ is presented for the more general Ricean channel. The symbols used in the table are defined as follows, $\theta_{\mathrm{r}} \triangleq \tan ^{-1}\left(\Re(r) / \Im(r), \sigma_{\mathrm{r}}^{2} \triangleq 2\left(\sigma_{h}^{2} s_{m}^{2}+\sigma_{n}^{2}\right), I_{0}\right.$ is the Modified Bessel function of the first type and zero order, and $\mathcal{I}_{\theta}$ is a nonlinear function defined in [34]. Moreover, the notation $(\cdot)^{\dagger}$ indicates that the detector is independent of the channel type. As can be noted from the table, the NC detector is the only detector that does not require CSI-I, but requires CSI-S. The CO detector has the lowest complexity, does not require CSI-S, but it requires accurate CSI-I. The $\mathrm{CO}$ detector design is also independent of the channel model. The AC-O has generally the highest complexity due to $\mathcal{I}_{\theta}$, and it requires CSI-I/S. The AC-S has lower complexity than the AC-O, but also requires both CSI-I/S. Finally, the AC-H is the detector with the lowest CSI requirements, which is $\alpha$, it has low complexity, and it is independent of the channel model. Consequently, the AC-H provides the best compromise in terms of complexity and BER performance, and hence it is considered in this work.

\section{WORK MOTIVATION AND CONTRIBUTION}

As can be noted from the surveyed literature, using higher order modulation combined with OFDM may have several advantages for OWC. However, the spectral efficiency, PAPR, phase noise, I/Q imbalance and CSI estimation errors are among the main limitations. Therefore, we consider in this work using $\mathrm{AC}$ detection, also known as semi-coherent detection [34], [35], [41] for OWC to jointly resolve some of the main limitations inherent to conventional optical OFDM systems. The AC detection relaxes the requirements for the channel estimation process, enables low complexity blind channel estimation and PAPR reduction, and has immunity to phase noise and I/Q imbalance. Moreover, OFDM spectral efficiency can be enhanced by using the IDCT and DCT for data modulation and demodulation, respectively, where using the DCT with one-dimensional data symbols enables avoiding the Hermitian symmetry constraint [43]. OFDM with DCT/IDCT will be denoted as C-OFDM, while conventional OFDM using the Fourier transform will be denoted as F-OFDM.

Although the AC detection has been considered in [34], [35], [41], to the best of the authors' knowledge, there is no work reported in the literature that considers $\mathrm{AC}$ detection for OWC. Therefore, this work incorporates AC detection into OWC, evaluates the opportunities and limitations that may arise, and compares its performance with other conventional detection schemes. Moreover, we propose new efficient PAPR reduction and blind channel-amplitude estimation schemes that are specifically designed for AC detection. The main advantages of the proposed PAPR reduction scheme are the low complexity and the fact that it does not require mapping side information to be communicated to the receiver. The proposed channel estimator can blindly and efficiently extract the channel-amplitudes, which are required for the AC detector to recover the information symbols. The obtained results corroborate that $\mathrm{AC}$ detection can be considered as an efficient detection scheme for OWC because it can significantly simplify the transmitter and receiver complexity, and offer high robustness for systems with severe phase noise.

\section{E. PAPER ORGANIZATION}

The rest of the paper is organized as follows. Section II presents the transmitted and received signal models, the channel model, spectral efficiency, and the proposed blind channel estimator. The proposed PAPR reduction scheme is presented in Section III. Section IV evaluates the complexity of the proposed system and compares it to conventional systems. Finally, the numerical results and conclusion are given in Sections V and VI, respectively.

\section{AMPLITUDE-COHERENT DETECTION FOR OPTICAL OFDM}

When AC detection is adopted, the data symbols should be modulated using unipolar $M$-ary ASK (MASK) [35]. Therefore, each data symbol $s$ is selected uniformly from a one-dimensional unipolar constellation $\mathbf{d}=\left[d_{0}\right.$, $\left.d_{1}, \ldots, d_{M_{A}-1}\right]$. Generating an OFDM signal using ASK symbols can be performed efficiently using the IDCT at the transmitter side [43]-[51]. By noting that the data and transform matrix elements have real values, then the IDCT output is real as well, and hence, the Hermitian symmetry constraint is not required for C-OFDM. However, the IDCT output is not necessarily positive, and thus, DCOOFDM or ACO-OFDM can be used [13] to generate strictly positive OFDM signals. Nevertheless, ACO-OFDM is considered in this work. Because Hermitian symmetry is not required for C-OFDM, the number of modulated subcarriers in an $N$-point IDCT is $N / 2$, because only odd subcarriers are modulated to enable asymmetric clipping operation without distorting the OFDM signal. Therefore, the IDCT output can be described by

$$
\mathbf{x}=\mathbf{T}^{-1} \tilde{\mathbf{s}}
$$

where $\tilde{\mathbf{s}}=\left[0, s_{0}, 0, s_{1}, \ldots, s_{N-2}, 0\right]$ is the zero-padded data symbols vector, and $\mathbf{T}$ is the $N \times N$ unitary DCT matrix with elements,

$$
T_{i, k}=\sqrt{\frac{2}{N}} \beta_{i} \cos \left(\frac{\pi i(2 k+1)}{2 N}\right),\{i, k\} \in\{0,1, \ldots, N-1\}
$$




$$
\beta_{i}= \begin{cases}\frac{1}{\sqrt{2}}, & i=0 \\ 1, & i=1,2, \ldots, N-1 .\end{cases}
$$

The complete C-OFDM symbol $\tilde{\mathbf{x}}$ is formed by adding the prefix and suffix guard bands [51].

In OWC, the channel coherence time is typically much longer than the symbol period, and hence, the channel can be considered constant over at least one symbol duration [52]. Therefore, the received signal after prefiltering, removing the guard intervals, and applying the DCT can be expressed as

$$
\tilde{\mathbf{r}}=\mathbf{H} \tilde{\mathbf{s}}+\tilde{\mathbf{n}}
$$

where $\tilde{\mathbf{r}}=\left[r_{0}, r_{1}, \ldots, r_{N-1}\right], \tilde{\mathbf{n}}=\left[n_{0}, n_{1}, \ldots, n_{N-1}\right]$ is the AWGN vector where $n_{i}$ is a complex Gaussian random variable with zero mean and $\sigma_{n}^{2}$ variance, $\mathbf{H}=$ $\left[\operatorname{diag}\left\{H_{0}, H_{1}, \ldots, H_{N-1}\right\}\right]$ is a diagonal matrix that represents the channel coefficients over the $N$ subcarriers. Given that the channel is flat [13], which is justified later in this section, then $H_{0}=H_{1}=\cdots=H_{N-1} \triangleq h$, and the received signal after dropping the unmodulated even subcarriers can be expressed as

$$
\mathbf{r}=h \mathbf{s}+\mathbf{n}
$$

where $\{\mathbf{r}, \mathbf{s}, \mathbf{n}\}$ are $(N / 2) \times 1$ vectors, $N$ is the total number of subcarriers.

Several models have been considered in the literature to represent the channel gain $|h|^{2} \triangleq \omega$, however, the doubly-stochastic scintillation Gamma-Gamma distribution has gained a wide acceptance for modeling atmospheric turbulence of FSO links [8], [53] and hence, it will be considered in this work. In the Gamma-Gamma model, the received intensity is considered as the product of two independent Gamma random variables, which represent the irradiance fluctuations arising from large and small-scale turbulence. The probability density function (PDF) of the Gamma-Gamma is given in [8, eq. (2)]. Given that $\omega$ is available at the receiver, the data symbols can be efficiently extracted using an $\mathrm{AC}$ detector by equalizing the energy of $r$, i.e., $|r|^{2}$, using $\omega$. Then, a simple threshold detector can be used, which corresponds to the heuristic AC detector [35].The data symbols are then extracted using on of the detectors listed in Table 1. However, the heuristic AC detector will be adopted in this work [34], [35], [41].

Although flat fading has been widely adopted to model OWC channels [52], the channel might become frequencyselective when the nonlinearity of optoelectronic devices is considered [52], [54]-[57]. Optoelectronic devices have a particular range where the frequency response can be considered flat. The flat range where the frequency response is flat mostly depends on the device characteristics and driving current. Some high quality devices such as the OSRAMs PL450B [54] can provide a near-flat bandwidth of about $1 \mathrm{GHz}$. In the case that higher transmission bandwidth is desired, or the design aims at using low-cost devices that do not have large flat range, then pre-distortion and pre-leveling techniques can be used to linearize the spectrum [58]-[61].

\section{A. SPECTRAL EFFICIENCY}

Based on the modulation type, three different OFDM configurations can be obtained. For fair comparison, we assume that all systems use ACO-OFDM. The three types are:

1) If two-dimensional modulation schemes such as quadrature amplitude modulation (QAM) are used, then the OFDM can be generated by applying the inverse fast Fourier transform (IFFT) at the transmitter. Given that the OFDM symbols consists of $N$ subcarriers produced using $N$-point IFFT, and $N_{Q}$ is the effective number of modulated subcarriers, then the spectral efficiency is given by

$$
\eta_{Q} \triangleq \frac{N_{Q}}{B_{Q}} \log _{2}\left(M_{Q}\right)
$$

where $M_{Q}$ is the modulation order and $B_{Q}$ is the bandwidth. For F-OFDM [44],

$$
\begin{aligned}
B_{Q} & =\frac{N+1}{T} \\
& \approx \frac{N}{T}, N \gg 1
\end{aligned}
$$

where $T$ is the symbol period, which can be normalized to one. For ACO-OFDM, only the odd subcarriers transmit data symbols, and the data vector should be Hermitian symmetric. Therefore, $N_{Q}=N / 4$, and

$$
\eta_{Q}=\frac{1}{4} \log _{2}\left(M_{Q}\right)
$$

2) If bipolar ASK ( $\overline{\mathrm{ASK}})$ is used, then additional spectral efficiency can be achieved by using the C-OFDM. By noting that the IDCT has subcarrier spacing of $1 / 2 T$ and the Hermitian symmetry constraint is waived, the number of modulated subcarriers $N_{\bar{A}}=N / 2$, and the bandwidth [44]

$$
\begin{aligned}
B_{\bar{A}} & =\frac{N+3}{2 T} \\
& \approx \frac{N}{2 T}, N \gg 3 .
\end{aligned}
$$

Therefore, the spectral efficiency

$$
\eta_{\bar{A}}=\log _{2}\left(M_{\bar{A}}\right)
$$

where $M_{\bar{A}}$ is the modulation order of the $\overline{\mathrm{ASK}}$ symbols.

3) For unipolar ASK (ASK) symbols that can be used for $\mathrm{AC}$ detection, the number of modulated subcarriers $N_{A}=N / 2$, and the bandwidth is similar to the $\overline{\mathrm{ASK}}$ case. Therefore,

$$
\eta_{A}=\log _{2}\left(M_{A}\right)
$$

where $M_{A}$ is the modulation order of the unipolar symbols.

For the three systems to have equal spectral efficiency, i.e., $\eta_{Q}=\eta_{\bar{A}}=\eta_{A}$, then the modulation orders should be selected such that

$$
M_{\bar{A}}=M_{A}=\sqrt[4]{M_{Q}} .
$$


Under the equal spectral efficiency constraint, each of the three configurations may have different modulation order, and hence different receiver complexity, BER, PAPR, and sensitivity to RF imperfections. For example, using 2 - $\overline{\mathrm{ASK}}$ is more desirable than a 16-QAM because it offers advantages in all aspects. The ASK can use the same modulation order, and hence, $\overline{\text { ASK }}$ will have better BER performance, but it will have higher receiver complexity due to the channel estimation needed for the coherent detection, and PAPR reduction process. Moreover, $\overline{\mathrm{ASK}}$ is more sensitive to RF impairments.

\section{B. BIND CHANNEL ESTIMATION}

CSI estimation is necessary to recover the information symbols coherently. In most practical systems, pilot symbols are ingrained within the data sequence $\mathbf{s}$ to enable the estimation of $h$. In flat fading channels, the estimation error can be reduced by averaging over multiple pilot symbols. Nevertheless, pilot symbols do not carry information and hence, they deteriorate the spectral efficiency.

When AC detection is adopted, the detector requires the knowledge of $\omega$ to extract the data symbols reliably, i.e., it does not require the channel phase. Using the maximum likelihood principle, the estimator can be formulated as

$$
\hat{\omega}=\arg \max _{\omega} f_{\mathbf{Y}}\left(y_{1}, y_{2}, \ldots, y_{\frac{N}{2}} \mid \omega\right)
$$

where $y_{l} \triangleq\left|r_{l}\right|^{2}$, and $f_{\mathbf{Y}}\left(y_{1}, y_{2}, \ldots, y_{\frac{N}{2}} \mid \omega\right)$ is the joint probability distribution function (PDF) of the received energies over the observation window $\frac{N}{2}$. By noting that $y_{1}, y_{2}, \ldots, y_{L}$ conditioned on $\omega$ are independent, then $f_{\mathbf{Y}}\left(y_{1}, y_{2}, \ldots, y_{L} \mid \omega\right)=\prod_{l=1}^{L} f_{Y}\left(y_{l} \mid \omega\right)$. As the natural logarithm (ln) is monotonic, then computing the log of all terms does not affect the maximization process. Thus

$$
\hat{\omega}=\arg \max _{\omega} \sum_{l=1}^{N / 2} \ln f_{Y}\left(y_{l} \mid \omega\right) .
$$

Because $\left[y_{1}, y_{2}, \ldots, y_{L}\right]$ are not identically distributed due to their dependence on the data symbols $s_{i}$, then using the Chain Rule we obtain,

$$
\hat{\omega}=\arg \max _{\omega} \sum_{l=1}^{N / 2} \ln \left[\sum_{s_{l}} f_{Y_{l}}\left(y_{l} \mid \omega, s_{l}\right) \operatorname{Pr}\left(s_{l}\right)\right] .
$$

By noting that $f_{Y_{l}}\left(y_{l} \mid \omega, s_{l}\right)$ has a conditional non-central Chisquared $\operatorname{PDF}, \operatorname{Pr}\left(s_{l}\right)=1 / M_{A}$, and after dropping the constant terms (16) can be written as

$$
\hat{\omega}=\arg \max _{\omega} \sum_{l=1}^{N / 2} \ln \left[\sum_{s_{l}} \mathrm{e}^{-\gamma\left(\omega E_{l}+y_{l}\right)} I_{0}\left(2 \gamma \sqrt{\omega y_{l} E_{l}}\right)\right]
$$

where $E_{l}=s_{l}^{2}$ and $\gamma=1 / 2 \sigma_{n}^{2}$. As can be noted from (17), solving the maximization problem is intractable, thus, a suboptimal estimation will be derived. As depicted in Appendix I, a suboptimal estimator can be expressed as

$$
\hat{\omega}=\frac{1}{2 L} \sum_{l \in \mathbb{L}}\left|r_{l}\right|^{2}
$$

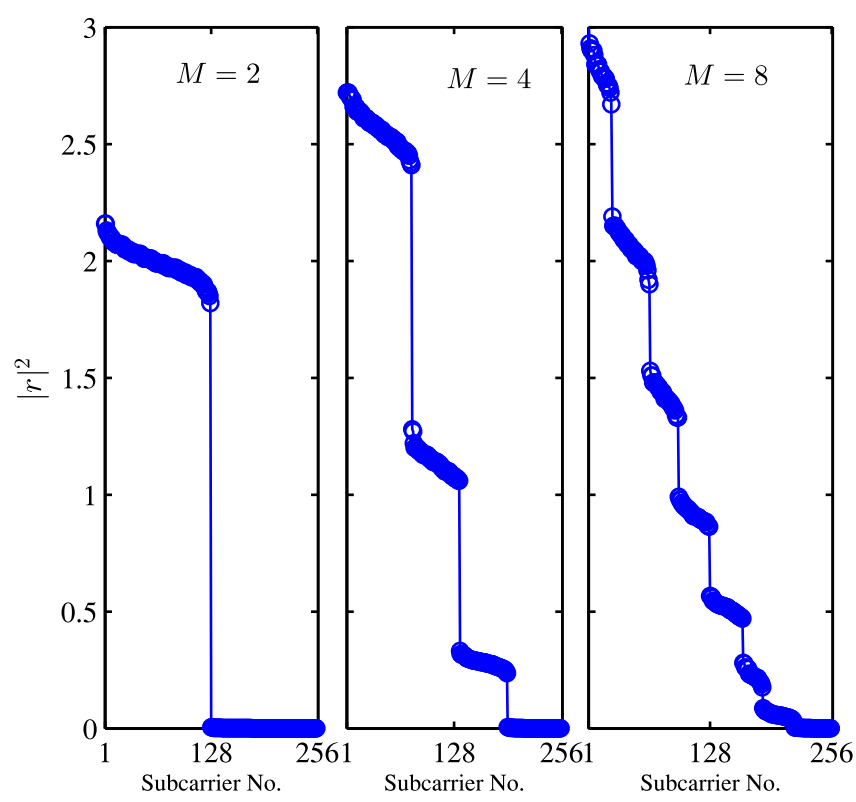

FIGURE 1. An example for the sorted energies for $M=2,4$, and $8, S N R=30 \mathrm{~dB}$.

where $\mathbb{L}$ is the set of indices that correspond to the case where $s_{l}=d_{M_{A}-1}$. Such estimator can be realized by noting that when all data symbols in $\mathbf{s}, s_{0}, s_{1}, \ldots, s_{\frac{N}{3}-1}$, are equally probable, then the average number of symbols with a particular amplitude is $N /\left(2 M_{A}\right)$. For example, in the binary case $M_{A}=2, d_{0}=0, d_{1}=\sqrt{2}$, thus on average, there are $N / 4$ symbols modulated using $d_{0}$ and $N / 4$ symbols using $d_{1}$. At high signal to noise ratios (SNRs), the energies $\left|r_{i}\right|^{2}$ that correspond to $d_{1}$ symbols are most likely to be larger than the energies for $d_{0}$ symbols. Consequently, choosing $L \leq N / 4$ samples with the maximum amplitudes among the $N / 2$ modulated subcarriers implies that we roughly manage to identify $L$ subcarriers whose data symbols are $d_{1}$. Fig. 1 shows an example of the received samples $\left|r_{i}\right|^{2}, i=1,2, \ldots, 256$, after being sorted for $M=2,4$, and 8 . The same approach can be applied for the case where $M_{A}>2$. However, if $L$ is not large enough to produce accurate estimates, we can extend the process to exploit more samples. For example, assuming that $M_{A}=4$, then we compute and sort $\left|r_{i}\right|^{2}$ for all received samples. Then, the samples are divided in three groups each of which has $N / 8 \triangleq L$ samples, where the first $L$ samples, denoted as $\mathbb{L}_{1}$, correspond to constellation point $d_{1}$, and so on. Therefore,

$$
\hat{\omega}=\frac{1}{M_{A}-1} \sum_{m=1}^{M_{A}-1}\left(\frac{1}{L d_{m}^{2}} \sum_{l \in \mathbb{L}_{m}}\left|r_{l}\right|^{2}\right) .
$$

The bias test for the derived estimator can be evaluated as

$$
\begin{aligned}
B_{\omega} & =\mathrm{E}[\hat{\omega} \mid \omega]-\omega \\
& =\mathrm{E}\left[\frac{1}{L E} \sum_{l \in \mathbb{L}}\left|r_{l}\right|^{2}\right]-\omega
\end{aligned}
$$




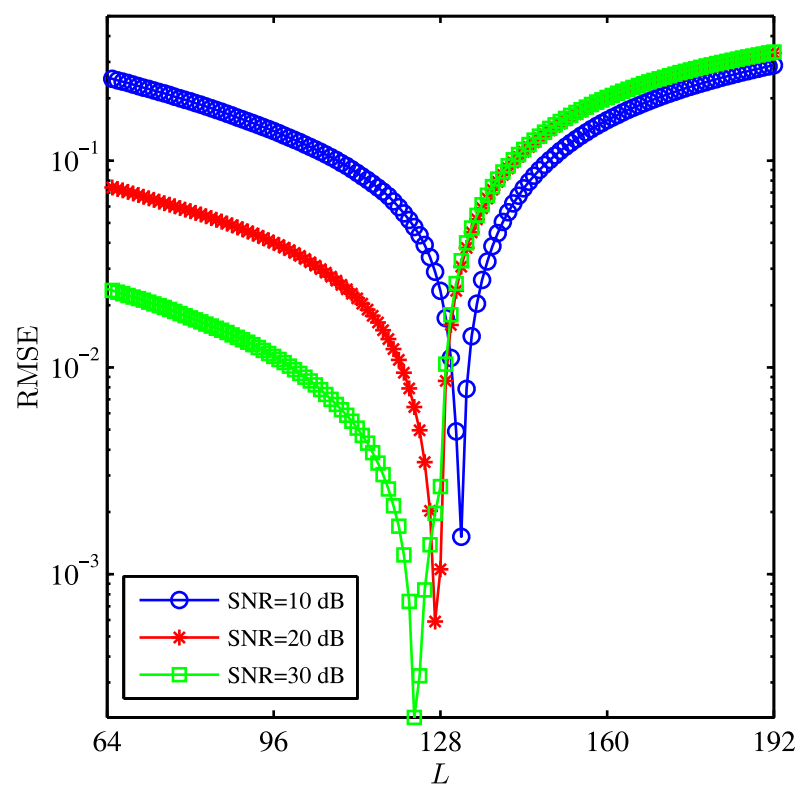

FIGURE 2. RMSE of the proposed channel estimator versus $L$ for $N=512, M=2$, and SNRs of 10,20 , and $30 \mathrm{~dB}$.

$$
=\frac{\omega}{L E} \sum_{l \in \mathbb{L}} \mathrm{E}\left[E_{l}\right]+\frac{1}{L E} \sum_{l \in \mathbb{L}} \sigma_{n, l}^{2}-\omega .
$$

Therefore, the bias depends on the accuracy of choosing $E_{l}$. Given that $E_{l}=E$, the bias reduces to

$$
B_{\omega}=\frac{1}{L E} \sum_{l \in \mathbb{L}} \sigma_{n, l}^{2} \text {. }
$$

At high SNR, $\sigma_{n}^{2} \ll 1$, or when $L \gg 1$, the bias is reduced significantly, which gives $B_{\omega} \approx 0$.

The mean-squared error (MSE) of the proposed estimator can be derived as

$$
\begin{aligned}
\mathrm{MSE} & =E\left[(\hat{\omega}-\omega)^{2}\right] \\
& =E\left[\hat{\omega}^{2}\right]-2 E[\hat{\omega} \omega]+E\left[\omega^{2}\right]
\end{aligned}
$$

By substituting the expression of $\hat{\omega}$ and expanding the resultant formula, it can be noted that several terms would result correspond to the product of multi correlated random variables with ordered statistics. Therefore, deriving the expectation of such cases is intractable, and thus we resort to Monte Carlo simulation to evaluate the MSE.

Fig. 2 shows the root mean-squared error (RMSE) of the proposed channel estimator for $N=512, M=2$, and SNRs of 10,20 , and $30 \mathrm{~dB}$. As can be noted from the figure, choosing the optimum value of $L$ is critical for the estimator to offer reliable channel estimates.

\section{PAPR REDUCTION USING RANDOM BIT-FLIPPING ALGORITHIM}

PAPR is one of the main limitations for OFDM systems, and it is more critical for unipolar ASK constellations because

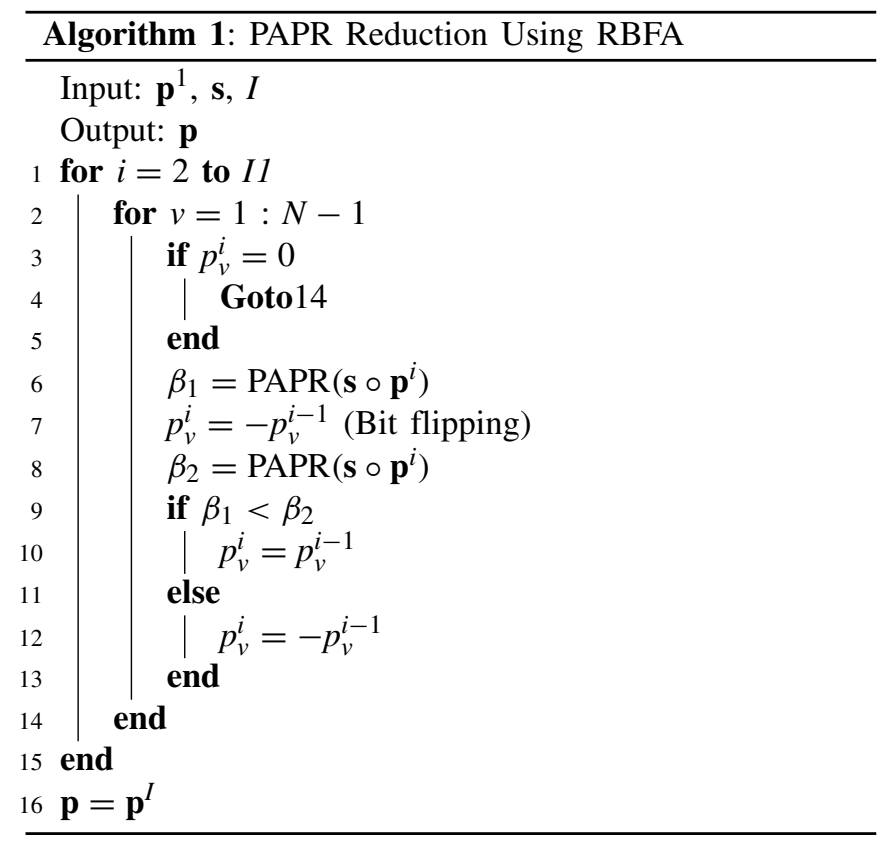

all symbols have the same polarity. The PAPR can be reduced by changing the phase of the data symbols using a predetermined sequence [25]-[27]

$$
\mathbf{p}=\left[p_{0}, p_{1}, \ldots, p_{N-1}\right], p_{i} \in\{1,0,-1\}
$$

Thus, the signal applied to the IDCT is $\mathbf{g}=\mathbf{s} \circ \mathbf{p}$, where ' $\circ$ ' denotes the Hadamard product. Generating the optimum phase sequence requires $2^{N / 2}$ trials, which is prohibitively expensive. Therefore, we propose a suboptimal heuristic algorithm, Algorithm 1, to compute $\mathbf{p}$ efficiently. Initially, a random version of $\mathbf{p}$ is generated offline, denoted as $\mathbf{p}^{1}$. If no additional iterations are desired, then $\mathbf{g}=\mathbf{s} \circ \mathbf{p}^{1}$, otherwise, Algorithm 1 is called to optimize $\mathbf{p}^{1}$ iteratively. In each iteration, the PAPR of the OFDM symbol is tested given $\mathbf{p}^{i}$, and then it is computed again after flipping the polarity of the first bit in $\mathbf{p}^{i}$, the final bit polarity is the one that provides less PAPR. Once the bit value is decided, it remains fixed to the end of the iteration. The process is repeated for all other bits in $\mathbf{p}^{i}$ using the same approach. Therefore, Algorithm 1 is called the random bit-flipping algorithm (RBFA), and it can be noted that it is a greedy algorithm within each iteration. The same process is repeated for all consequent iterations. In addition to its low complexity, the proposed algorithm does not require to send any information to the receiver about the sequence $\mathbf{p}$, because the phase information is irrelevant for AC detection.

It is worth noting that there are several other bit flipping algoritms as reported in [24] and the references listed therein. Nevertheless, the proposed RBFA is fundamentally different because it does not have constraints on the bits to be flipped, and does not have to be accompanied with an error correction scheme to mitigate the BER degradation caused by the bit flipping process. 


\section{COMPLEXITY ANALYSIS}

The complexity of the proposed C-OFDM and conventional F-OFDM is mostly determined by the modulation/demodulation operations, PAPR reduction scheme, CSI estimation, and data detection. Therefore, each of these subsystems is discussed and compared to conventional FOFDM. The computational complexity is derived assuming that the FFT and fast cosine transform (FCT) are adopted. The computational complexity is evaluated in terms of the number of required mathematical operations such as complex multiplication $\left(C_{\times}\right)$, complex addition $\left(C_{+}\right)$, real multiplications $\left(R_{\times}\right)$and real additions $\left(R_{+}\right)$, etc.

\section{A. MODULATION-DEMODULATION}

\section{1) F-OFDM:}

- The OFDM symbol generation at the transmitter and data extraction at the receiver requires the computation of an IFFT and FFT, respectively. Computing an $N$-point FFT or IFFT using efficient algorithms such as the radix-2 Cooley-Tukey algorithm requires $C_{\mathrm{M}}=\mathcal{N} / 2$ and $C_{\mathrm{A}}=\mathcal{N}$, where $\mathcal{N}=N \log _{2} N$. By noting that each complex multiplication is equivalent to three real multiplications and three real additions, and each complex addition is equivalent to two real additions, the total number of real multiplication and addition operations required for the IFFT plus FFT is $R_{\mathrm{M}}=3 \mathcal{N}$ and $R_{\mathrm{A}}=10 \mathcal{N}$, respectively.

- After FFT at the receiver, the data subcarriers are extracted where even and Hermitian symmetry subcarriers are discarded. The complexity of this operation is generally negligible because the subcarrier allocation is fixed.

- The subcarriers that carry information will be equalized with a zero-forcing using an estimated version of the channel coefficient. The complexity of the channel coefficient estimation is discussed in Section II-B. The information symbols can be extracted from the soft samples after equalization using a maximum likelihood detector (MLD). The complexity of the equalization process and MLD for a QAM symbol requires $R_{\mathrm{M}}=6 M_{Q}$ and $R_{\mathrm{A}}=5 M_{Q}$ per symbol. Therefore, the total complexity for the MLD process for all subcarriers is significant, particularly for large modulation orders.

\section{2) C-OFDM:}

- For the FCT, all operations are real where $R_{\mathrm{M}}=$ $0.5 \mathcal{N}$ and $R_{\mathrm{A}}=1.5 \mathcal{N}-N+1$ [44]. Therefore, the total IFCT plus FCT operations are $R_{\mathrm{M}}=\mathcal{N}$ and $R_{\mathrm{A}}=3 \mathcal{N}-2 N+2$. Consequently, the number of real multiplications for the F-OFDM is three times larger than the C-OFDM, and the real additions are more than seven times larger.
- After the FCT, the odd subcarriers are extracted, and similar to the F-OFDM case, the complexity of this step is negligible.

- The energy for each sample is then computed, which has $R_{\mathrm{M}}=2$ and $R_{\mathrm{A}}=1$ for each data subcarrier.

- The equalization process requires only one real division, which is simpler than F-OFDM case. The complexity of the channel estimation is given in Section II-B.

- Unlike coherent QAM, AC detection can be performed efficiently using the heuristic AC detector [34], [35], [41].

It is also worth noting the complexity difference between the detectors of the two configurations. In the case that the two configurations are using the same modulation order, then the complexity of the two detectors is generally equivalent. However, under equal spectral efficiency constraints, the modulation order of the F-OFDM is much larger, and hence, the complexity of the MLD is much higher as well.

\section{B. PAPR REDUCTION}

The proposed PAPR reduction complexity can be summarized as follows:

1) Computing $\mathbf{g}=\mathbf{p}^{1} \circ \mathbf{s}$, the complexity of this process is negligible because it only requires flipping the signs of the non-zero elements in s. If signed integer representation is used, then flipping the phase requires simply inverting one bit.

2) For each non-zero bit, flip the bit value and compute the IFCT using the updated bit value. Given that $x_{m}^{i} \mid p_{v}^{i}$ is the $m$ th sample at the IFCT output computed using $p_{v}^{i}$ during the $i$ th iteration, then $x_{m}^{i+1} \mid p_{v}^{i+1}$ can be computed as

$$
x_{m}^{i+1}\left|p_{v}^{i+1}=x_{m}^{i}\right| p_{v}^{i}-p_{v}^{i} \times 2 \cos \left(\frac{\pi v[2 m+1]}{2 N}\right)
$$

which is due to the fact that $p_{v}= \pm 1$. Moreover, the term

$$
2 \cos (\pi v[2 m+1] / 2 N)
$$

can be computed one time off-line, and the value of $p_{v}$ can be used to control the signs of its elements. As a result, for each non-zero bit we need to compute $N$ real subtractions, and thus, the number of real subtractions for each OFDM symbol is given by

$$
R_{-}=\frac{(I-1)}{4} N^{2} \text {. }
$$

However, no multiplications are required. It is worth noting that capturing the signal peak accurately requires oversampling the IFCT output, which can be achieved using a zero-padded data vector, which also increases the size of the IFCT. Nevertheless, the IFCT 
complexity scales linearly with the oversampling factor $Q$ due to the sparseness of the oversampled data vector.

3) Computing the PAPR typically requires calculating

$$
\frac{\max \left\{x_{m}^{2}\right\}}{\sum_{m} x_{m}^{2}}, \forall x_{m} \neq 0 .
$$

Because the data samples are real, each squaring process requires only one real multiplication. However, it should be noted that after zero-clipping, the OFDM signal becomes positive, further to being real. Consequently, the PAPR reduction can be achieved by minimizing the peak-to-average amplitude ratio (PAAR), which is given by

$$
\text { PAAR }=\frac{\max \left\{x_{m}\right\}}{\sum_{m} x_{m}} .
$$

Computing PAAR (27) requires much lower computational complexity when compared to the conventional PAPR (26), because no multiplication operations are required. Monte Carlo simulation results confirmed that using either metric would result in the same PAPR reduction value.

The complexity of PAPR reduction of the F-OFDM is determined by the algorithm used. For example, if the partial transmit sequence (PTS) is used, then we have to search for the best phase sequence that will be used, and in each search iteration a $Q N$-point IFFT should be computed $V$ times, where $V$ is the number of partitions, typically more than 16 . Given that the phase sequence used is binary, then at least $V+1$ iterations are needed. Consequently, the complexity of the proposed PAPR reduction algorithm is substantially less complex than the algorithms used for F-OFDM with QAM symbols.

\section{CHANNEL ESTIMATION}

As can be noted from Section II-B, CSI estimation can be divided into three main steps:

1) Compute $\left|r_{i}\right|^{2} \triangleq \gamma_{i}$ for all data samples at the DCT output, however, these value are needed for the detection process, and hence their complexity is already considered as a part from the detection process.

2) Select the maximum $L$ values from $\left\{\gamma_{1}, \gamma_{3}, \ldots, \gamma_{N-1}\right\}$, which presumably correspond to $L$ samples where symbol $d_{M_{A}-1}$ is transmitted. The value of $L$ should be selected to minimize the mean-square error (MSE). Given that the transmitted symbols are equiprobable, then the average number of symbols whose value is $d_{M_{A}-1}$ would be $N /\left(2 M_{A}\right)$. Consequently, if $L \gg N /\left(2 M_{A}\right)$, then several samples where $d_{i} \neq d_{M_{A}-1}$ will be included, which may deteriorate the MSE, and using $L \ll N /\left(2 M_{A}\right)$ implies that several useful samples are not utilized, which may affect the MSE as well. Therefore, the size of $L$ should be optimized to minimize the MSE.
Nevertheless, Monte Carlo simulation results revealed that using $L \geq 16$ is sufficient to provide BERs that are equivalent to those obtained using perfect channel estimates. Therefore, we consider a fixed $L$ in this work while deriving the optimum $L$ will be considered in our future work. Furthermore, because we do not strictly require the maximum $L$ elements, lower and faster algorithms can be developed. For example, the $L$ samples can be selected by comparing $\gamma_{i}$ to a certain threshold, which may significantly reduce the time and space requirements. The complexity of the sorting process is usually evaluated in terms of time and space, and thus, cannot be evaluated in terms of number of mathematical operations. A special case of interest is for the 2-ASK scenario, where it can be demonstrated that $\omega$ can be estimated accurately using all data samples. Therefore, a significant complexity reduction can be obtained by eliminating the search for the maximum $L$ values. Such result is obtained because the samples modulated using $d_{0}$ have very limited effect on the estimation accuracy, even if they are included in the estimation process.

3) Compute the estimated $\hat{\omega}$ as described in Section II.B which has $R_{+}=2 L+1, R_{\times}=2$, and $R_{\div}=2$, where $R \div$ denotes the real divisions.

Therefore, the overall computational complexity of the proposed channel estimation algorithm is fairly low. Comparing the proposed algorithm to the conventional leastsquare estimation using pilot symbols, it can be noted that choosing the maximum $L$ symbols is the only additional operations required. However, the proposed algorithm is blind because no pilot symbols are required.

\section{NUMERICAL RESULTS}

The section presents the numerical results for an OFDM based OWC system where the number of subcarriers $N=64$ and the three system models are considered, which are the C-OFDM with ASK and $\overline{\text { ASK }}$ modulation, and F-OFDM using QAM. The results are generated given that all systems have the same spectral efficiency, and hence, two groups of modulation orders are used $\mathbf{m}_{1}=[2,2,16]$ and $\mathbf{m}_{2}=[4$, $4,256]$, where the elements of $\mathbf{m}$ represent the modulation orders of the $M_{A}, M_{\bar{A}}$ and $M_{Q}$, respectively. All the considered systems are implemented as ACO-OFDM, and thus, the data is transmitted on the odd subcarriers while even subcarriers are nulled. For the F-OFDM, the Hermitian symmetry is considered, while it is not applied for the C-OFDM because the data and transform are real. The negative part of the signals is clipped at the zero level, and different clipping levels are used for the positive signals. The $\overline{\mathrm{ASK}}$ and QAM symbols are recovered using a coherent MLD while ASK symbols are recovered using the heuristic AC detector. A flat fading channel with Gamma-Gamma distribution is considered where $\alpha=8$ and $\beta=6$, and thus, the Rytov variance (scintillation index) $\sigma_{\mathrm{R}}=0.3125>0.3$, which corresponds 


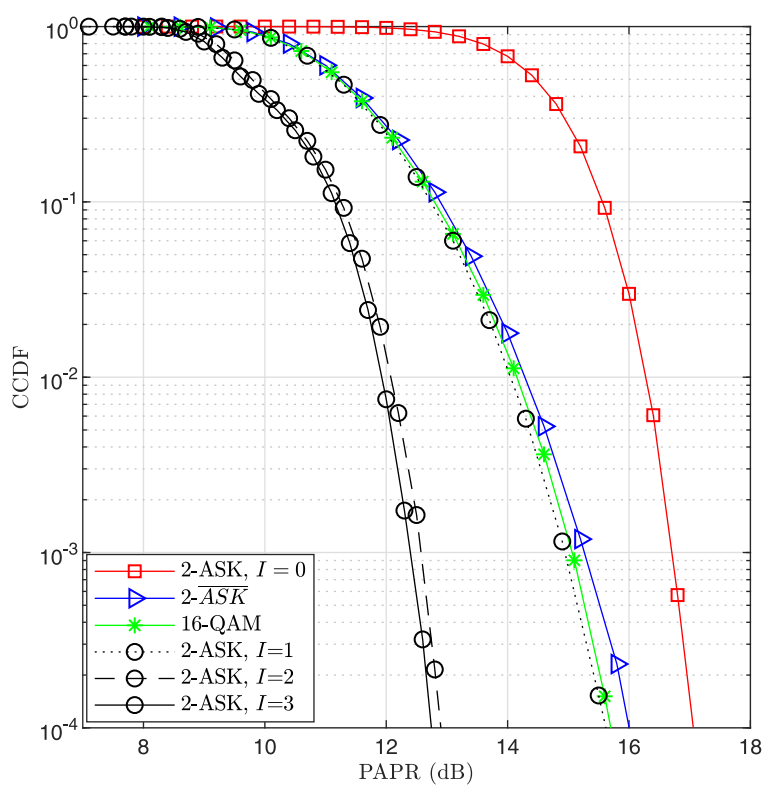

FIGURE 3. CCDF for the considered modulation schemes, the ASK is considered with and without PAPR reduction.

to a moderate turbulence environment. Monte Carlo simulation is performed using $10^{5}$ realizations for each simulation point. The detection for all types of modulation schemes is performed while considering perfect CSI, unless stated otherwise.

Fig. 3 shows the complementary cumulative distribution function (CCDF) of the PAPR for the considered systems using $\mathbf{m}_{1}$ scenario. The PAPR is measured after zeroclipping, which actually increases the PAPR for all systems by $3 \mathrm{~dB}$. The 2 -ASK is considered with and without the proposed PAPR reduction algorithm while the other modulation schemes are considered without PAPR reduction. As can be noted from the figure, the PAPR of the 2-ASK without PAPR reduction is higher than all the other considered systems, which makes it the most susceptible to the system nonlinearity. Using Algorithm 1 with one iteration reduced the PAPR by about $1 \mathrm{~dB}$ at CCDF of $10^{-4}$, which makes it comparable to the PAPR of 2- $\overline{\mathrm{ASK}}$ and 16-QAM cases. Using one additional iteration may provide an additional 3 $\mathrm{dB}$ of PAPR reduction as compared to the case with a single iteration. However, the PAPR reduction that can be achieved by increasing the number of iterations to three provided only an additional $0.25 \mathrm{~dB}$. Therefore, using $I>2$ provides negligible additional PAPR reduction.

Fig. 4 shows the BER that can be achieved at the receiver by applying different detection methods using $\mathbf{m}_{1}$ and $\mathbf{m}_{2}$. The transmitter is assumed to have linear amplifier and LED, and hence, positive clipping is not considered. For the $\mathbf{m}_{1}$ scenario, the $2-\overline{\mathrm{ASK}}$ offers the best performance as expected, followed by the 2-ASK, and then 16-QAM. The 2-ASK has moderate BER where there is about $6 \mathrm{~dB}$ loss at $10^{-4}$ as compared to the 2- $\overline{\mathrm{ASK}}$, while the 2-ASK and 16-QAM have comparable BER. For the $\mathbf{m}_{2}$ scenario, the same trend

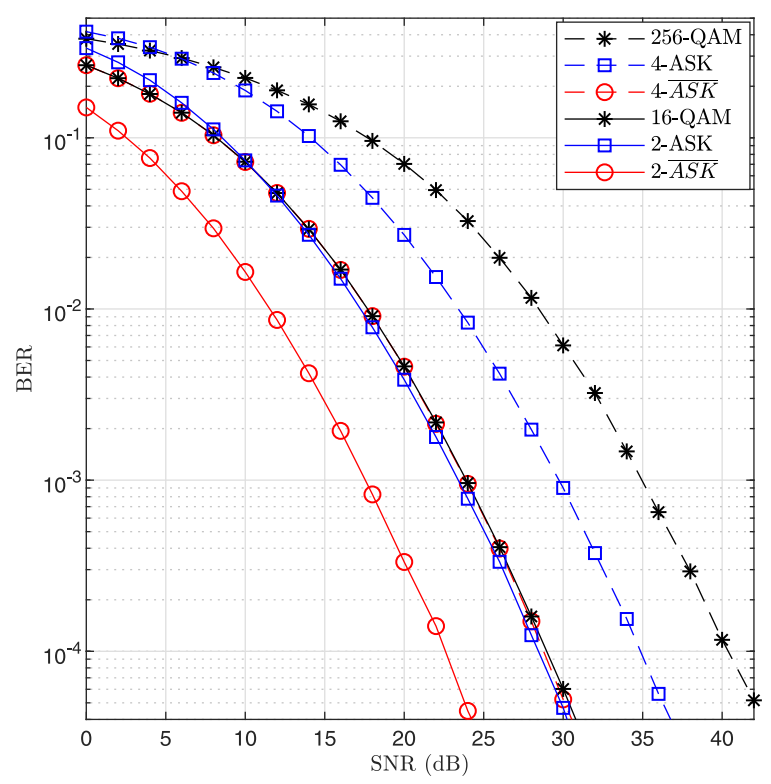

FIGURE 4. BER for different detection methods and different values of $\boldsymbol{M}$.

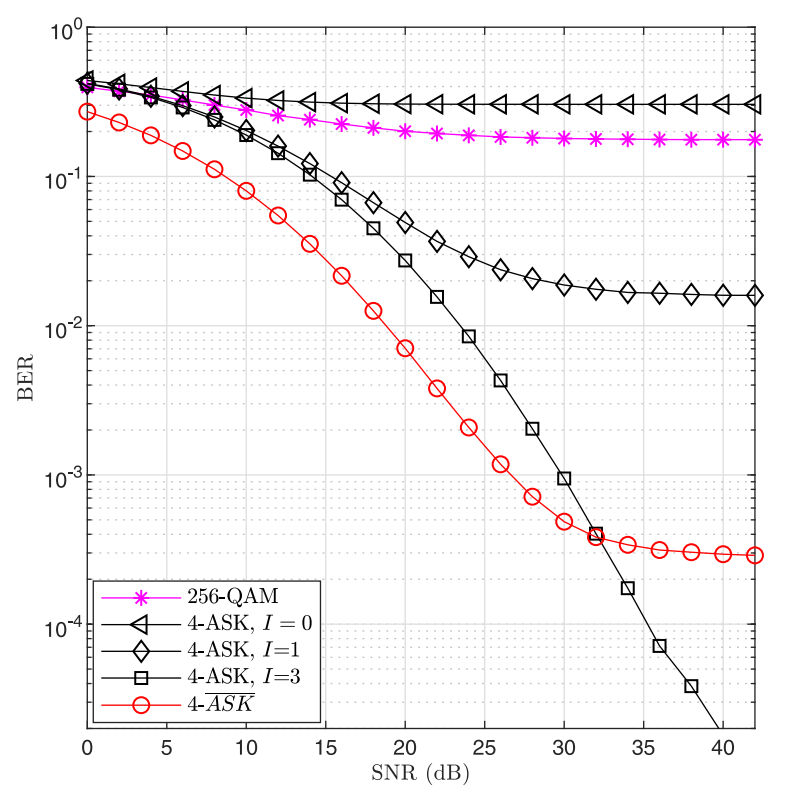

FIGURE 5. BER versus SNR for the ASK with PAPR reduction and variying number of iterations, compared to the $\overline{\mathrm{ASK}}$ and QAM without PAPR reduction. The positive clipping factor $\mu_{c}=1.5$.

is maintained, except that the 4-ASK outperforms the 256QAM by about $6 \mathrm{~dB}$ at BER of $10^{-4}$. It is also worth noting that the 2-ASK, 4- $\overline{\mathrm{ASK}}$ and 16-QAM have comparable BER.

Fig. 5 presents the BER versus SNR (dB) for the considered schemes using $\mathbf{m}_{2}$ where a fixed positive clipping level $\mu_{c}=1.5$ is applied. The ASK is considered with PAPR reduction using different number of iterations, and the case where $I=0$ indicates that no PAPR reduction is utilized. The figure clearly shows that 4-ASK without PAPR reduction and 256-QAM perform very poorly with high BER floors for the considered SNR range. The $4-\overline{\mathrm{ASK}}$ performs 


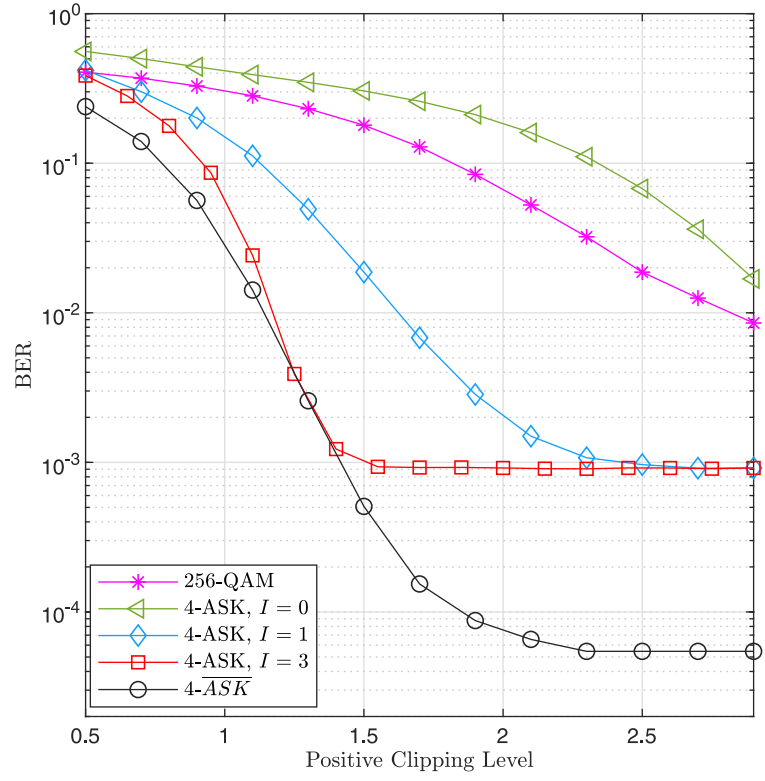

FIGURE 6. BER versus the clipping factor $\mu_{c}$ of the ASK using a varying number of iterations compared to the $\overline{A S K}$ and QAM without PAPR reduction, $\mathrm{SNR}=30$.

well at low SNRs because the performance in this range is dominated by the AWGN, however at high SNRs, the BER is dominated by the inter-carrier interference (ICI) caused by the clipping, and thus, an error floor starts to appear for SNR $>35 \mathrm{~dB}$. The impact of PAPR reduction on ASK is demonstrated for $I=1$ and 3 , where the case of $I=1$ shows some improvement, but an error floor can still be observed at BER of $\sim 1.8 \times 10^{-2}$, where the symbol ' $\sim$ ' denotes the weak approximation. It is also worth noting that the PAPR using $I=1$ is similar to the $4-\overline{\mathrm{ASK}}$, nevertheless, the 4- $\overline{\mathrm{ASK}}$ has better BER because it is less sensitive to ICI. Using $I=3$ with 4-ASK eliminates the error floor, and thus, outperforms the 4- $\overline{\mathrm{ASK}}$ at high SNRs.

Fig. 6 shows the BER versus the clipping level $\mu_{c}$ of $\mathbf{m}_{2}$ scenario using a fixed SNR $=30 \mathrm{~dB}$. The SNR is generally selected high so that the performance is mostly determined by the ICI. As can be noted from the figure, using low clipping values deteriorates the BER for all schemes significantly. However, the 4-ASK with $I=0$ and 256QAM are the most sensitive to the clipping level. The 4-ASK using $I=3$ and 4- $\overline{\text { ASK }}$ exhibit equivalent BER for $\mu_{c} \sim 1.3$ because the $4-\overline{\mathrm{ASK}}$ does not use PAPR reduction. The BER of the 4-ASK using $I=3$ becomes independent of the clipping for $\mu_{c} \gtrsim 1.6$, while it is about 2.3 for the 4 - $\overline{\mathrm{ASK}}$ case. It is worth noting that the 4-ASK outperforms the 4$\overline{\mathrm{ASK}}$ for $\mathrm{SNR}>30 \mathrm{~dB}$ when $\mu_{c} \sim 1.3$, the results are omitted to avoid crowding the figure.

Fig. 7 shows the effect of the phase noise on the considered detection schemes using $\mathbf{m}_{2}$. The phase noise is modeled as a Tikhonov random variable [34, eq. (19)], with variance $\sigma_{\phi}^{2}=\left[1^{\circ}, 3^{\circ}, 5^{\circ}, 7^{\circ}\right]$. The BER of the ASK is represented by a single curve because AC detection is immune to phase noise. The BER for the 256-QAM is presented only for $\sigma_{\phi}^{2}=1^{\circ}$ because the BER deteriorates significantly even for such low phase noise scenarios. The 4- $\overline{\mathrm{ASK}}$ is more immune than the 256-QAM, yet there is a significant degradation for $\sigma_{\phi}^{2} \geq 5^{\circ}$. The figure also shows the ASK performance using the proposed blind channel estimation. As can be noted from the figure, using $L=16$ is sufficient to provide near-optimal BER for SNR $\lesssim 33 \mathrm{~dB}$ while using $L=1$ caused a $3 \mathrm{~dB}$ BER degradation. At $\mathrm{SNR} \approx 40 \mathrm{~dB}$, using $L=1$ provides more accurate channel estimates because the $L=16$ case may consist of data symbols with the incorrect constellation point, which implies that $L$ should be selected dynamically based on the system and channel parameters.

\section{A. OPPORTUNITIES AND CHALLENGES}

As demonstrated by the obtained results, using IDCT/DCT with unipolar ASK and AC detection outperforms conventional OFDM with QAM in the context of OWC. Moreover, it provides the system designer additional degrees of freedom that can be used to design efficient PAPR reduction and channel estimation algorithms. The system immunity against phase noise makes it more robust than bipolar ASK with coherent detection at high SNRs.

The main limitation of unipolar ASK is the small Euclidean distance between the ASK constellation points, which makes it prone to higher error rates at low SNRs, as compared to ASK. Such performance is achieved because BER performance at low SNR $s$ is dominated by AWGN, even in the presence of phase noise, which manifests itself as $6 \mathrm{~dB}$ degradation in terms of BER. Therefore, more research is needed to mitigate this limitation. For the proposed subsystems, i.e., the PAPR reduction and channel estimation algorithms, the main focus of this work is the complexity reduction, thus, these techniques have the potential to be improved. For example, using a fixed number of samples for the channel estimation process might lead to a poor estimation accuracy if the data is not uniformly distributed. Therefore, the number of samples used for channel estimation should be dynamically selected based on the received OFDM block. Such goal can be achieved by modifying the channel estimation algorithm to dynamically identify the gap between different constellation levels as shown in Fig. 1. The PAPR algorithm has several benefits in terms of complexity and data side information. Nevertheless, the random phase sequence can be actually designed to carry some information, not necessarily with the same reliability as the main data stream. Therefore, the system can support two data streams with unequal error protection properties.

\section{CONCLUSION AND FUTURE WORK}

This paper presented the design and performance evaluation of an OFDM based OWC system using AC detection. By exploiting the facts that $\mathrm{AC}$ detection requires real data at the transmitter, and it does not require phase information at the receiver, an efficient transmitter/receiver design with PAPR reduction and channel estimation techniques was proposed. The modulator was designed using the IDCT, and hence the 


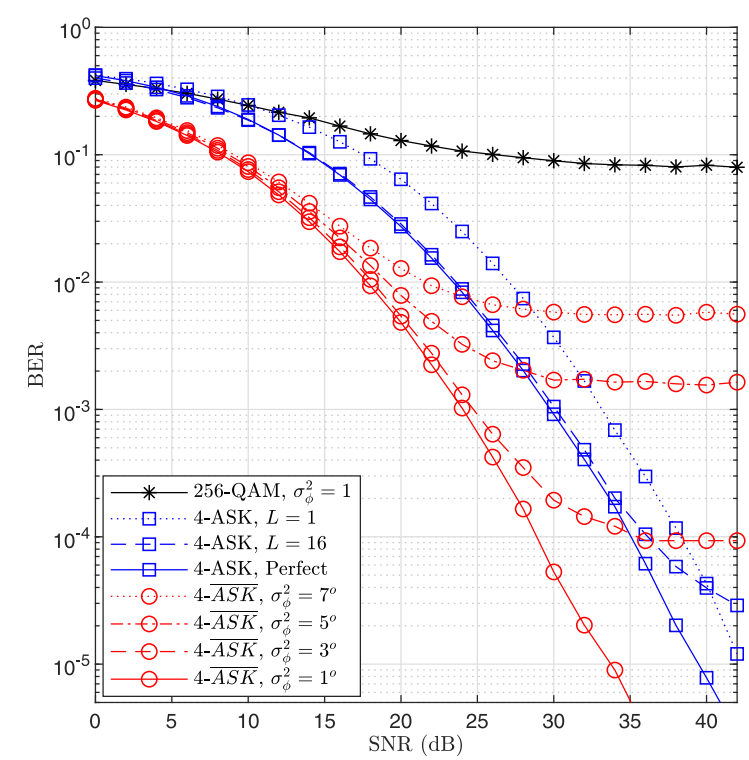

FIGURE 7. The effect of phase noise on the considered detection mehods for different values of phase noise variance $\sigma_{\phi}^{2}, M=4$, and no clipping.

Hermitian symmetry constraint was omitted, which improved the spectral efficiency of the system. The proposed PAPR reduction algorithm starts by randomly changing the phase of the transmitted data sequence, and then improve the PAPR iteratively. Consequently, the algorithm complexity was minimized, and no mapping information should be exchanged between the transmitter and receiver. The complexity analysis and simulation results confirmed the efficiency of the proposed PAPR reduction algorithm. The proposed channel estimation algorithm resorted to the flatness of the OWC channel to recognize the symbols with the maximum modulation orders, and used them as virtual pilots. Consequently, the channel estimates were obtained using a large number of virtual pilots, which provided near perfect CSI. The BER of the proposed system was evaluated and compared to other OFDM based systems using coherent detection in the presence of phase noise, and the obtained results confirmed the robustness of the $\mathrm{AC}$ detector. Consequently, integrating $\mathrm{AC}$ detection with $\mathrm{OWC}$ has several desirable features as compared to conventional detection schemes.

Our future work will focus on evaluating the impact of various synchronization errors on the system performance to assess its tolerance to such errors. Moreover, new synchronization algorithms will be specifically designed to suit the proposed system. The ACD should be tested with single carrier systems...

\section{APPENDIX I: CHANNEL ESTIMATOR DERIVATION}

For cases where $s_{l}=d_{0}=0$, the received $r_{l} \mid s_{l}=0$, $\forall l=[1,2, \ldots, N / 2]$ are independent of $\omega$, and can be separated from the summation in (17), which after dropping the constant terms gives

$$
\hat{\omega}=\arg \max _{\omega} g(\omega)
$$

where

$$
\begin{aligned}
g(\omega)=\sum_{l=1}^{N / 2} \ln \left[f_{Y_{l}}\left(y_{l} \mid d_{0}\right) \operatorname{Pr}\left(d_{0}\right)\right. & \\
& \left.+\sum_{s_{l} \in \overline{\mathbf{d}}} f_{Y_{l}}\left(y_{l} \mid \omega, s_{l}\right) \operatorname{Pr}\left(s_{l}\right)\right]
\end{aligned}
$$

Therefore,

$$
\begin{aligned}
g(\omega) & =\sum_{l=1}^{N / 2} \ln \left[\frac{\mathrm{e}^{-\gamma y_{l}}}{M_{A}}+\frac{1}{M_{A}} \sum_{s_{l} \in \overline{\mathbf{d}}} \frac{I_{0}\left(2 \gamma \sqrt{\omega y_{l} E_{l}}\right)}{\mathrm{e}^{\gamma\left(\omega E_{l}+y_{l}\right)}}\right] \\
& =\sum_{l=1}^{N / 2} \ln \left[\mathrm{e}^{-\gamma y_{l}}+\sum_{s_{l} \in \overline{\mathbf{d}}} \frac{I_{0}\left(2 \gamma \sqrt{\omega y_{l} E_{l}}\right)}{\mathrm{e}^{\gamma\left(\omega E_{l}+y_{l}\right)}}\right] \\
& =\sum_{l=1}^{N / 2} \ln \left[1+\sum_{s_{l} \in \overline{\mathbf{d}}} \frac{I_{0}\left(2 \gamma \sqrt{\omega y_{l} E_{l}}\right)}{\mathrm{e}^{\gamma \omega E_{l}}}\right]
\end{aligned}
$$

Because the ln function is monotonic, then (30) can be simplified to

$$
\hat{\omega}=\arg \max _{\omega} \sum_{l=1}^{N / 2} \ln \left[\sum_{s_{l} \in \overline{\mathbf{d}}} \mathrm{e}^{-\gamma \omega E_{l}} I_{0}\left(2 \gamma \sqrt{\omega y_{l} E_{l}}\right)\right]
$$

where $\overline{\mathbf{d}}=\left[d_{1}, d_{2}, \ldots, d_{M_{A}-1}\right]$.

Because $g(\omega)$ is a concave down function, the solution for the estimator in (31) can be obtained as

$$
\hat{\omega}=\omega \mid\left(\frac{\partial}{\partial \omega} g(\omega)=0\right)
$$

where

$$
\frac{\partial}{\partial \omega} g(\omega)=\sum_{l=1}^{N / 2} \frac{\partial}{\partial \omega} \ln \left[\sum_{s_{l} \in \overline{\mathbf{d}}} \mathrm{e}^{-\gamma \omega E_{l}} I_{0}\left(2 \gamma \sqrt{E_{l} \omega y_{l}}\right)\right] .
$$

Although the derivative in (33) can be solved in a closedform, solving for $\omega$ is still intractable due to the Bessel function. Therefore, the Bessel function is approximated as $I_{0}(x) \approx \frac{e^{x}}{\sqrt{2 \pi x}}$, and thus substituting for $I_{0}(\cdot)$ gives

$$
\begin{aligned}
\frac{\partial}{\partial \omega} g(\omega) & \approx \sum_{l=1}^{N / 2} \frac{\partial}{\partial \omega} \ln \left[\frac{1}{2 \sqrt{\pi \gamma}} \sum_{s_{l} \in \overline{\mathbf{d}}} \frac{\mathrm{e}^{-\gamma\left(\omega E_{l}-2 \sqrt{E_{l} \omega y_{l}}\right)}}{\sqrt[4]{\omega y_{l} E_{l}}}\right] \\
& =\sum_{l=1}^{N / 2} \frac{\partial}{\partial \omega} \ln \left[\sum_{s_{l} \in \overline{\mathbf{d}}} \frac{\mathrm{e}^{-\gamma\left(\omega E_{l}-2 \sqrt{E_{l} \omega y_{l}}\right)}}{\sqrt[4]{\omega y_{l} E_{l}}}\right]
\end{aligned}
$$

However, for the binary case $\overline{\mathbf{d}}=[\sqrt{2}]$, or by considering that $s_{l}=d_{m} \forall l$, then $\left\{s_{l}, E_{l}\right\} \rightarrow\{s, E\}$, and

$$
\begin{aligned}
\frac{\partial}{\partial \omega} g(\omega) & =\sum_{l \in \mathbb{L}} \frac{\partial}{\partial \omega} \ln \left[\frac{\mathrm{e}^{-\gamma\left(\omega E-2 \sqrt{E \omega y_{l}}\right)}}{\sqrt[4]{\omega y_{l} E}}\right] \\
& =-\frac{L}{4 \omega}+\left(\sum_{l \in \mathbb{L}}\left[\frac{\gamma E y_{l}}{\sqrt{\omega E y_{l}}}-\gamma E\right]\right)
\end{aligned}
$$


Finally, by setting $\frac{\partial}{\partial \omega} g(\omega)=0$ and collecting the terms with respect to $\gamma$ gives

$$
-\frac{L}{4 \hat{\omega} \gamma}+E \sum_{l \in \mathbb{L}}\left[\frac{y_{l}}{\sqrt{\hat{\omega} E y_{l}}}-1\right]=0 .
$$

At high SNRs, $\gamma \gg 1$, thus, the term $\frac{L}{\gamma 4 \omega}$ becomes much smaller than the other terms, and hence it can be dropped without affecting the estimator accuracy. Therefore (36) can be simplified to

$$
\sum_{l \in \mathbb{L}} \frac{\sqrt{y_{l}}}{\sqrt{\hat{\omega} E}}-L=0
$$

which gives

$$
\sqrt{\hat{\omega}}=\frac{1}{\sqrt{E} L} \sum_{l \in \mathbb{L}} \sqrt{y_{l}}
$$

By noting the relation between $\sqrt{\hat{\omega}}$ and $\sqrt{y_{l}}$, then a low complexity heuristic estimator for $\hat{\omega}$ can be derived as

$$
\hat{\omega}=\frac{1}{E L} \sum_{l \in \mathbb{L}} y_{l} \text {. }
$$

\section{REFERENCES}

[1] K. Wang, Indoor Infrared Optical Wireless Communications: Systems and Integration. Boca Raton, FL, USA: CRC Press, 2019.

[2] Z. Ghassemlooy, L.-N. Alves, S. Zvanovec, and M.-A. Khalighi, Visible Light Communications: Theory and Applications. London, U.K.: Taylor \& Francis, 2019

[3] Z. Ghassemlooy, Optical Wireless Communications: System and Channel Modelling with MATLAB. Milton, ON, Canada: CRC Press, 2019.

[4] D. Tsonev et al., "A 3-Gb/s single-LED OFDM-based wireless VLC link using a Gallium nitride $\mu$ LED," IEEE Photon. Technol. Lett., vol. 26, no. 7, pp. 637-640, Apr. 2014.

[5] M. Esmail, A. Ragheb, H. Fathallah, and M. Alouini, "Experimental demonstration of outdoor 2.2 Tbps super-channel FSO transmission system," in Proc. IEEE Int. Conf. Commun. Workshops (ICC), Kuala Lumpur, Malaysia, 2016, pp. 169-174.

[6] J. Puschell and R. Bayse, "High data rate ultraviolet communication systems for the tactical battlefield," in Proc. Tactical Commun. Conf., Fort Wayne, IN, USA, 1990, pp. 253-267.

[7] S. Xiaobin et al., "71-Mbit/s ultraviolet-B LED communication link based on 8-QAM-OFDM modulation," Opt. Exp., vol. 25, no. 19, pp. 23267-23274, 2017.

[8] M. Khalighi and M. Uysal, "Survey on free space optical communication: A communication theory perspective," IEEE Commun. Surveys Tuts., vol. 16, no. 4, pp. 2231-2258, 4th Quart., 2014.

[9] Y. Wang et al., "Non-data-aided cycle slip self-correcting carrier phase estimation for QPSK modulation format of coherent wireless optical communication system," IEEE Access, vol. 7, pp. 110451-110462, 2019.

[10] P. Sahoo, Y. Prajapati, and R. Tripathi, "PPM- and GMSK-based hybrid modulation technique for optical wireless communication cellular backhaul channel," IET Commun., vol. 12, no. 17, pp. 2158-2163, Oct. 2018.

[11] C. Gong, K. Wang, Z. Xu, and X. Wang, "On full-duplex relaying for optical wireless scattering communication with on-off keying modulation," IEEE Trans. Wireless Commun., vol. 17, no. 4, pp. 2525-2538, Apr. 2018.

[12] J. Armstrong, "OFDM for optical communications," J. Lightw. Technol., vol. 27, no. 3, pp. 189-204, Feb. 01, 2009.

[13] S. Dissanayake and J. Armstrong, "Comparison of ACO-OFDM, DCO-OFDM and ADO-OFDM in IM/DD systems," J. Lightw. Technol., vol. 31, no. 7, pp. 1063-1072, Apr. 01, 2013.

[14] I. Lu, C. Yeh, D. Hsu, and C. Chow, "Utilization of 1-GHz VCSEL for 11.1-Gbps OFDM VLC wireless communication," IEEE Photon J., vol. 8, no. 3, pp. 1-6, Jun. 2016.
[15] J. He, J. He, and J. Shi, "An enhanced adaptive scheme with pairwise coding for OFDM-VLC system," IEEE Photon. Technol. Lett., vol. 30, no. 13, pp. 1254-1257, Jul. 01, 2018.

[16] Y. Yang, Z. Zeng, S. Feng, and C. Guo, "A simple OFDM scheme for VLC systems based on $\mu$-law mapping," IEEE Photon. Technol. Lett., vol. 28, no. 6, pp. 641-644, Mar. 15, 2016.

[17] T. Zhang, Y. Zou, J. Sun, and S. Qiao, "Improved companding transform for PAPR reduction in ACO-OFDM-based VLC systems," IEEE Commun. Lett., vol. 22, no. 6, pp. 1180-1183, Jun. 2018.

[18] X. Huang, J. Shi, J. Li, Y. Wang, and N. Chi, "A Gb/s VLC transmission using hardware preequalization circuit," IEEE Photon. Technol. Lett., vol. 27, no. 18, pp. 1915-1918, Sep. 15, 2015.

[19] Y. Wang, X. Huang, J. Zhang, Y. Wang, and N. Chi, "Enhanced performance of visible light communication employing 512-QAM NSC-FDE and DD-LMS," Opt. Exp., vol. 22, no. 13, pp. 15328-15334, 2014.

[20] D. Aller, D. Lamar, P. Miaja, J. Rodríguez, and J. Sebastián, "Taking advantage of the sum of the light in outphasing technique for visible light communication transmitter," IEEE $J$. Emerg. Sel. Topics Power Electron., early access, Jan. 10, 2020, doi: 10.1109/JESTPE.2020.2965774.

[21] Y. Cheong, X. Ng, and W. Chung, "Hazardless biomedical sensing data transmission using VLC," IEEE Sensors J., vol. 13, no. 9, pp. 3347-3348, Sep. 2013.

[22] M. Abadi, Z. Ghassemlooy, M. Khalighi, S. Zvanovec, and M. Bhatnagar, "FSO detection using differential signaling in outdoor correlated-channels condition," IEEE Photon. Technol. Lett., vol. 28 , no. 1, pp. 55-58, Jan. 01, 2016.

[23] S. Idris, A. Aibinu, G. Koyunlu, and J. Sanusi, "A survey of modulation schemes in visible light communications," in Proc. 3rd Int. Conf. Trends Electron. Informat. (ICOEI), Tirunelveli, India, 2019, pp. 1-7.

[24] Y. Liu, C. Chang, S. Lee, and M. Lin, "Deliberate bit flipping with error-correction for PAPR reduction," IEEE Trans. Broadcast., vol. 63, no. 1, pp. 123-133, Mar. 2017.

[25] M. Ahmed, S. Boussakta, A. Al-Dweik, B. Sharif, and C. Tsimenidis, "Efficient design of selective mapping and partial transmit sequence using T-OFDM," IEEE Trans. Veh. Technol., vol. 69, no. 3, pp. 2636-2648, Mar. 2020.

[26] E. Al-Dalakta, A. Al-Dweik, A. Hazmi, C. Tsimenidis, and B. Sharif, "PAPR reduction scheme using maximum cross correlation," IEEE Commun. Lett., vol. 16, no. 12, pp. 2032-2035, Dec. 2012.

[27] E. Al-Dalakta, A. Al-Dweik, A. Hazmi, C. Tsimenidis, and B. Sharif, "Efficient BER reduction technique for nonlinear OFDM transmission using distortion prediction," IEEE Trans. Veh. Technol., vol. 61, no. 5, pp. 2330-2336, Jun. 2012.

[28] W. Hu and D. Lee, "PAPR reduction for visible light communication systems without side information," IEEE Photon. J., vol. 9, no. 3, pp. 1-11, Jun. 2017.

[29] H. Zhang, Y. Yuan, and W. Xu, "PAPR reduction for DCO-OFDM visible light communications via semidefinite relaxation," IEEE Photon. Technol. Lett., vol. 26, no. 17, pp. 1718-1721, Sep. 01, 2014.

[30] T. Zhang, J. Zhou, Z. Zhang, Y. Lu, F. Su, and Y. Qiao, "Dimming control systems based on low-PAPR SCFDM for visible light communications," IEEE Photon. J., vol. 10, no. 5, pp. 1-11, Oct. 2018.

[31] W. Hu, "PAPR reduction in DCO-OFDM visible light communication systems using optimized odd and even sequences combination," IEEE Photon. J., vol. 11, no. 1, pp. 1-15, Feb. 2019.

[32] M. Obeed, A. Salhab, M. Alouini, and S. Zummo, "On optimizing VLC networks for downlink multi-user transmission: A survey," IEEE Commun. Surveys Tuts., vol. 21, no. 3, pp. 2947-2976, 3rd Quart., 2019.

[33] X. Yi et al., "Phase noise effects on phase-modulated coherent optical OFDM," IEEE Photon J., vol. 8, no. 1, pp. 1-8, Feb. 2016.

[34] M. Al-Jarrah, K.-H. Park, A. Al-Dweik, and M.-S. Alouni, "Error rate analysis of amplitude-coherent detection over Rician fading channels with receiver diversity," IEEE Trans. Wireless Commun., vol. 19, no. 1, pp. 134-147, Jan. 2020.

[35] A. Al-Dweik and Y. Iraqi, "Error probability analysis and applications of amplitude-coherent detection in flat Rayleigh fading channels," IEEE Trans. Commun., vol. 64, no. 5, pp. 2235-2244, May 2016.

[36] A. Saci, A. Al-Dweik, A. Shami, and Y. Iraqi, "One-shot blind channel estimation for OFDM systems over frequency-selective fading channels," IEEE Trans. Commun., vol. 65, no. 12, pp. 5445-5458, Dec. 2017. 
[37] K. Kiasaleh, "Receiver architecture for channel-aided, OOK, APDbased FSO communications through turbulent atmosphere," IEEE Trans. Commun., vol. 63, no. 1, pp. 186-194, Jan. 2015.

[38] J. Estrada-Jiménez, B. Guzmán, M. García, and V. Jiménez, "Superimposed training-based channel estimation for MISO opticalOFDM VLC," IEEE Trans. Veh. Technol., vol. 68, no. 6, pp. 6161-6166, Jun. 2019.

[39] L. Yang, J. Cheng, and J. Holzman, "Maximum likelihood estimation of the lognormal-Rician FSO channel model," IEEE Photon. Technol. Lett., vol. 27, no. 15, pp. 1656-1659, Aug. 2015.

[40] M. Uysal, F. Miramirkhani, O. Narmanlioglu, T. Baykas, and E. Panayirci, "IEEE 802.15.7r1 reference channel models for visible light communications," IEEE Commun. Mag., vol. 55, no. 1, pp. 212-217, Jan. 2017.

[41] L. Bariah, A. Al-Dweik, S. Muhaidat, Y. Iraqi, and M. Al-Mualla, "Performance analysis of semi-coherent OFDM systems with imperfect channel estimates," IEEE Trans. Veh. Technol., vol. 67, no. 11, pp. 10773-10787, Nov. 2018.

[42] M. Riediger, R. Schober, and L. Lampe, "Blind detection of onoff keying for free-space optical communications," in Proc. Can. Conf. Elect. Comput. Eng., Niagara Falls, ON, Canada, 2008, pp. 001361-001364.

[43] J. Zhou, Y. Qiao, Z. Cai, and Y. Ji, "Asymmetrically clipped optical fast OFDM based on discrete cosine transform for IM/DD systems," J. Lightw. Technol., vol. 33, no. 9, pp. 1920-1927, May 01, 2015.

[44] F. Xiong, "M-ary amplitude shift keying OFDM system," IEEE Trans. Commun., vol. 51, no. 10, pp. 1638-1642, Oct. 2003.

[45] M. Chafii, J. Coon, and D. Hedges, "DCT-OFDM with index modulation," IEEE Commun. Lett., vol. 21, no. 7, pp. 1489-1492, Jul. 2017

[46] P. Tan and N. Beaulieu, "A comparison of DCT-based OFDM and DFT-based OFDM in frequency offset and fading channels," IEEE Trans. Commun., vol. 54, no. 11, pp. 2113-2125, Nov. 2006.

[47] J. Yang, J. Lee, and S. Kim, "Channel optimized predistortion for self-heterodyne DCT-based OFDM systems," IEEE Trans. Consum. Electron., vol. 51, no. 3, pp. 770-775, Aug. 2005.

[48] C. He, A. Cao, L. Xiao, L. Zhang, P. Xiao, and K. Nikitopoulos, "Enhanced DCT-OFDM system with index modulation," IEEE Trans. Veh. Technol., vol. 68, no. 5, pp. 5134-5138, May 2019.

[49] F. Gao, T. Cui, A. Nallanathan, and C. Tellambura, "Maximum likelihood based estimation of frequency and phase offset in DCT OFDM systems under non-circular transmissions: Algorithms, analysis and comparisons," IEEE Trans. Commun., vol. 56, no. 9, pp. 1425-1429, Sep. 2008.

[50] W. Chin, "Maximization of effective signal power in DCT window for symbol time synchronization in optical fast OFDM," J. Lightw. Technol., vol. 31, no. 5, pp. 740-748, Mar. 01, 2013.

[51] N. Al-Dhahir, H. Minn, and S. Satish, "Optimum DCT-based multicarrier transceivers for frequency-selective channels," IEEE Trans. Commun., vol. 54, no. 5, pp. 911-921, May 2006.

[52] A. Al-Kinani, C. Wang, L. Zhou, and W. Zhang, "Optical wireless communication channel measurements and models," IEEE Commun. Surveys Tuts., vol. 20, no. 3, pp. 1939-1962, 3rd Quart., 2018.

[53] M. Uysal, J. Li, and M. Yu, "Error rate performance analysis of coded free-space optical links over gamma-gamma atmospheric turbulence channels," IEEE Trans. Wireless Commun., vol. 5, no. 6, pp. 1229-1233, Jun. 2006

[54] S. Viola, M. S. Islim, S. Watson, S. Videv, H. Haas, and A. E. Kelly, "15 Gb/s OFDM-based VLC using direct modulation of $450 \mathrm{GaN}$ laser diode," in Proc. Adv. Free Space Opt. Commun. Techn. Appl. III, vol. 10437. Warsaw, Poland, Oct. 2017, pp. E1-E7.

[55] A. Lorences-Riesgo et al., "200 G outdoor free-space-optics link using a single-photodiode receiver," J. Lightw. Technol., vol. 38, no. 2, pp. 394-400, Jan. 15, 2020.

[56] J. Holguin-Lerma, M. Kong, O. Alkhazragi, X. Sun, T. Ng, and S. Ooi, "480-nm distributed-feedback InGaN laser diode for 10.5-Gbit/s visible-light communication," Opt. Lett., vol. 45, no. 3, pp. 742-745, Feb. 2020.

[57] C. Lee et al., "Gigabit-per-second white light-based visible light communication using near-ultraviolet laser diode and red-, green-, and blue-emitting phosphors," Opt. Exp., vol. 25, pp. 17480-17487, Jul. 2017.

[58] L. Wei, C. Chow, C. Hsu, and C. Yeh, "Bidirectional visible light communication system using a single VCSEL with predistortion to enhance the upstream remodulation," IEEE Photon. J., vol. 10, no. 3, pp. 1-7, Jun. 2018.
[59] Y. Huang et al., "Blue laser diode based free-space optical data transmission elevated to 18 Gbps over 16 m," Sci. Rep., vol. 7, Sep. 2017, Art. no. 10478

[60] S. Liang, Z. Jiang, L. Qiao, X. Lu, and N. Chi, "Faster-than-nyquist precoded CAP modulation visible light communication system based on nonlinear weighted look-up table predistortion," IEEE Photon. J., vol. 10, no. 1, pp. 1-9, Feb. 2018.

[61] W.-C. Wang, H.-Y. Wang, and G.-R. Lin, "Ultrahigh-speed violet laser diode based free-space optical communication beyond $25 \mathrm{Gbit} / \mathrm{s}$," Sci. Rep., vol. 8, Sep. 2018, Art. no. 13142.

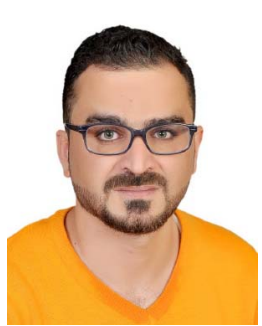

MOHAMMAD A. AL-JARRAH (Member, IEEE) received the B.Sc. and M.S. degrees in electrical engineering/wireless communications from the Jordan University of Science and Technology in 2011. He is currently pursuing the Ph.D. degree with the Department of Electrical and Electronic Engineering, University of Manchester, U.K., where he is currently a Marie Curie early stage Researcher. From 2017 to 2019, he had been working as a Lab Instructor with Khalifa University, UAE. His research interests include distributed decision fusion systems, statistical signal processing, target tracking in wireless sensor networks, RFID communications, cooperative spectrum sensing, free-space optical communications, cooperative communications, and backhauling and cellular planning for $5 \mathrm{G}$ cellular networks.

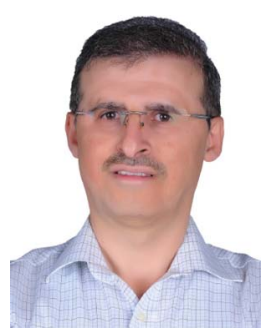

ARAFAT AL-DWEIK (Senior Member, IEEE) received the Ph.D. degree (Magna Cum Laude) in electrical engineering from Cleveland State University, Cleveland, OH, USA, in 2001. He was with Efficient Channel Coding, Inc., Cleveland, OH, USA, from 1999 to 2001. From 2001 to 2003, he was the Head of the Department of Information Technology, Arab American University, Palestine. Since 2003, he has been with the Department of ECE, Khalifa University, UAE. He joined the University of Guelph, ON, Canada, as an Associate Professor from 2013 to 2014. He serves as an Editor for the IEEE TRANSACTIONS ON VEHICUlar TECHNOLOGY and IET Communications.

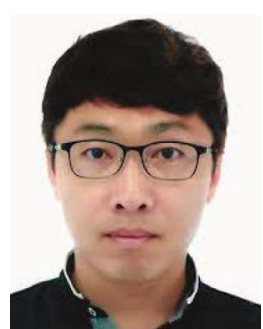

KI-HONG PARK (Senior Member, IEEE) received the B.Sc. degree in electrical, electronic, and radio engineering from Korea University, Seoul, South Korea, in 2005, and the M.S. and Ph.D degrees from the School of Electrical Engineering, Korea University in 2011. In April 2011, he joined the King Abdullah University of Science and Technology as a Postdoctoral Fellow. Since December 2014, he has been working as a Research Scientist of electrical engineering with the Division of Computer, Electrical, Mathematical Science and Engineering. His research interests are in communication theory and its application to the design and performance evaluation of wireless communication systems, underwater visible light communication, optical wireless communications, unmanned aerial vehicle communication, and physical layer secrecy.

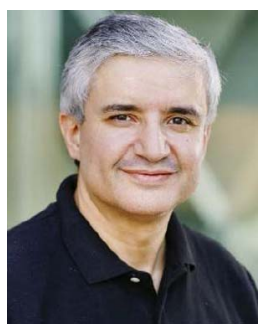

MOHAMED-SLIM ALOUINI received the Ph.D. degree in electrical engineering from the California Institute of Technology, Pasadena, CA, USA, in 1998. In 2009, he joined the King Abdullah University of Science and Technology, Thuwal, Saudi Arabia, as a Professor of electrical engineering. He served as a Faculty Member with the University of Minnesota, Minneapolis, MN, USA, and Texas A\&M University at Qatar, Education City, Doha, Qatar. His current research interests include the modeling, design, and performance analysis of wireless communication systems. 